\title{
Mythos
}

Rivista di Storia delle Religioni

$10 \mid 2016$

Varia

\section{Das Orakelwesen im Alten Ägypten}

Oracles in Ancient Egypt

\section{Alexandra von Lieven}

\section{(Q) OpenEdition}

\section{Journals}

\section{Edizione digitale}

URL: http://journals.openedition.org/mythos/400

DOI: $10.4000 /$ mythos.400

ISSN: 2037-7746

\section{Editore}

Salvatore Sciascia Editore

\section{Edizione cartacea}

Data di pubblicazione: 1 décembre 2016

Paginazione: 17-30

ISBN: 978-88-8241-476-4

ISSN: 1972-2516

\section{Notizia bibliografica digitale}

Alexandra von Lieven, «Das Orakelwesen im Alten Ägypten », Mythos [En ligne], 10 | 2016, mis en ligne le 24 septembre 2019, consulté le 28 septembre 2019. URL : http://journals.openedition.org/mythos/ 400 ; DOl : 10.4000/mythos.400 


\section{Das Orakelwesen im Alten Ägypten*}

\section{Alexandra von Lieven}

\section{Zusammenfassung}

Aus dem Alten Ägypten sind zahlreiche unterschiedliche divinatorische Techniken bezeugt, mit deren Hilfe der Wille der Götter, die juristische Wahrheit oder die Zukunft bestimmt werden konnten. Am weitaus besten dokumentiert sind dabei verschiedene Orakelarten, die die Götter unmittelbar in Form ihrer Kultstatuen oder ihrer heiligen Tiere involvierten. Solche Orakel konnten sowohl öffentlichen wie privaten Charakter haben. Der Beitrag diskutiert die verschiedenen Spielarten und inre jeweilige Anwendung.

\section{Schlüsselworte}

Orakel • Divination - Kultbarke

\section{Keywords}

Oracle • divination • sacred bark

\section{Abstract}

From Ancient Egypt, many different divinatory techniques are attested. They were used to determine the will of the gods, the juridical truth or the future. Best documented are different types of oracles which involved the gods directly, either in form of their statues or in form of sacred animals. Such oracles could both have an official or a more private character. The article discusses the different types and their respective uses.

«Da ich viele Leute angetroffen habe, die sich selbst durch Divination betrogen fühlen, erschien es mir rasch notwendig, damit keine Gefahr aus ihrer Torheit erwachsen möge, hiermit deutlich alle Leute aufzufordern, von diesem irreführenden Aberglauben Abstand zu nehmen. Deshalb laßt keinen Menschen durch Orakel, das bedeutet, mit Hilfe schriftlicher Dokumente, die vermeintlich in die Gegenwart der Gottheit gelegt werden, auch nicht durch die Prozession von Kultbildern oder derartigen Scharlatanerien, vorgeben, er hätte Wissen um das Übernatürliche, oder behaupten, die Verborgenheit künftiger Ereignisse zu kennen. Laßt auch nicht einen Menschen sich Verfügung verschaffen über solche, die danach verlangen oder auf irgendeine Art darauf antworten. Wenn irgend eine Person ausfindig gemacht wird, die diesem Gewerbe angehört, laßt ihn sicher sein, daß er strenger Bestrafung überantwortet wird...»${ }^{1}$

$\mathrm{B}$ ei diesem Text handelt es sich nicht etwa um ein Dokument aus christlicher Zeit, sondern um ein Dekret des römischen Präfekten für Ägypten unter dem Kaiser Septimius Severus, Q. Aemilius Saturninus aus dem Jahr 199 n. Chr. Es zeigt in aller Deutlichkeit, daß die traditionelle Technik des Prozessionsorakels, das in Ägypten seit dem Neuen Reich, also ca. 1500 v. Chr., gut faßbar ist, noch am Vorabend des 3. Jh. n. Chr. ausgiebig praktiziert wurde, ja offenbar so ausgiebig, daß die römischen Autoritäten, die ja auch sonst divina-

* Dieser Beitrag wurde während eines Heisenberg-Stipendiums (Geschäftszeichen Li 1846/1-1) verfaßt, für das ich der Deutschen Forschungsgemeinschaft (DFG) herzlich danken möchte.

1 Ritner 1993, 218 (pYale 299), Jördens 2008b, 445. 
torische Praktiken ${ }^{2}$ gerne kontrollierten, um politischen Unruhen vorzubeugen, sich davon bedroht sahen.

Die in dem zitierten Dekret angedeutete Orakelform läßt sich durch bildliche, textliche und archäologische Quellen durch die Zeiten näher präzisieren. Prozessionen, in deren Verlauf ein Kultbild aus seinem Tempel herausgetragen und z.B. besuchsweise zu einem anderen Tempel einer anderen Gottheit verbracht wurde, gehörten zu den wichtigsten und verbreitetsten Formen ägyptischer Festrituale. Dabei wurde das Kultbild auf einer Barke mit Tragstangen ${ }^{3}$, in einem Tragsessel oder in der Spätzeit auch auf einem Wagen ${ }^{4}$ transportiert. Auf den Barken und Wagen befanden sich Schreine zur Aufnahme der Statue, wobei die Schreine auf den Barken zusätzlich durch ein weißes Tuch partiell verhüllt waren. Lediglich bei den Tragsesseln, die vorwiegend im Kult vergöttlichter Könige belegt sind, war die darin thronende Statue ohne weiteres sichtbar ${ }^{5}$. Die Sichtbarkeit oder Unsichtbarkeit hängt sicherlich mit dem Grad an Transzendenz der göttlichen Wesenheit zusammen. Die Barken oder Sessel wurden auf den Schultern der Priester der jeweiligen Gottheit getragen. Bei Wagen dürften sie von den Priestern gezogen worden sein. Über diese Transportform liegen leider weniger Belege vor, auch ist kein eindeutig damit zu verbindender Fall eines Orakels bekannt. Man sollte die Möglichkeit jedoch im Auge behalten.

Welches Gedränge um die Götterbarke geherrscht haben dürfte, mag der ethnographische Vergleich mit ganz ähnlich gearteten Prozessionsfesten im heutigen hinduistischen Indien besonders gut illustrieren. Daß man sich dies auch für Ägypten entsprechend vorstellen muß, legt etwa die Lehre des Ani nahe, die aus der Ramessidenzeit stammt, als diese Art der religiösen Feier in ihrer Hochblüte stand ${ }^{6}$. Dort wird der Zögling ermahnt, sich nicht so dicht an die Barke mit dem Götterbild heranzudrängeln, da dies dem Gott unangenehm sei. Faktisch dürfte aber gerade dies in der Realität das erklärte Ziel vieler Gläubiger gewesen sein - war doch das Prozessionsfest die einzige Möglichkeit, dem eigentlichen Kultbild aus dem Tempelinneren so nahe zu kommen, wie sonst nie. Selbst wenn man es nicht sehen konnte, garantierte dennoch die physische Nähe die Erhörung der Bitten in besonderer Weise. Es ist also nur folgerichtig, daß diese Prozessionen auch zur Orakelbefragung dienten.

2 Die hier besprochenen Orakel sind zwar die gemeinhin bekannteste divinatorische Technik des Alten Ägypten, aber bei Weitem nicht die einzig bezeugte. Für einen Gesamtüberblick s. von Lieven 1999. Diese ihrer Intention nach zum Abfassungszeitpunkt vollständige Belegsammlung wäre inzwischen durch Neufunde bzw. seither publiziertes Material erheblich zu erweitern. Als wichtigste Ergänzungen mit auch typologisch neuem Material seien hier nur Demichelis 2002 und QuACK 2006 genannt. S. weiterhin den Beitrag von J. Quack in vorliegendem Band mit weiterer Literatur.

3 Zur Ikonographie solcher Barken s. KarLshausen 2009.

4 Der Gebrauch von Wagen für religiöse Prozessionen wird durch Herodot II 63 bezeugt, man wird sie sich wohl so vorzustellen haben, wie die Wagen bei el AMIR 1948, Pl. XVII und das Vergleichsstück Leipzig 1830 (LANDesmuseum WürtTemberg 2007, 294-295), die allerdings im Zusammenhang von Bestattungsprozessionen heiliger Stiere stehen, bei denen keine Orakel stattfanden. Man beachte, daß alle diese Wagen eigentlich Barken auf Rädern sind, dasselbe Phänomen findet sich auch in Darstellungen aus Privatgräbern der Spätzeit (s. z.B. Cherpion, Corteggiani, Gout 2007, 134-135). Darin zeigt sich, daß für die Ägypter eigentlich die Barke das einzig korrekte Fortbewegungsmittel für Götter und Verstorbene darstellte.

5 Für eine einschlägige Darstellung s. etwa Foucart 1932, Pl. XXVIII aus dem Grab des Amunmose (TT 19).

6 QuACK 1994, 108-109 (B 20,13-14), 134-137 (D 5,5-7), 175-176, 311-312. 
Dazu gab es jedoch mehrere verschiedene Techniken ${ }^{7}$. Einerseits konnte man eben im Rahmen solcher Prozessionen vor die Gottheit treten und offenbar mündlich eine Frage stellen. Diese wurde dann durch nickende Bewegungen bzw. das Voranschreiten oder Zurückweichen des Kultbildes bzw. der Barke beantwortet. Auf derartige Techniken könnten spätzeitliche Personennamen vom Typ $\breve{C} t-h r-n-N N$ referieren, also „Das Gesicht des NN hat gesprochen“8. Dabei dürfte mit dem Gesicht nicht so sehr das des ja im Schrein verborgenen Kultbildes, als das der Barke gemeint sein, denn die Götterbarken zeichneten sich dadurch aus, daß sie an Bug und Heck den Kopf der jeweiligen Gottheit aufwiesen.

Diese Technik eignete sich gut, um Fragen von einer gewissen öffentlichen Relevanz zu klären, im Privatbereich insbesondere juristische Querelen in Eigentumsfragen oder zur Schuldfeststellung. Dies geht denn auch als Hauptaufgabe solcher Orakel aus den darüber erhaltenen Niederschriften hervor ${ }^{9}$. Außerdem ist das Barkenorakel im Neuen Reich die Hauptform des Orakels in Staatsangelegenheiten, bei denen der König höchst selbst die Gottheit in wichtigen politischen oder administrativen Belangen befragte.

Andere Fragen freilich gingen niemand etwas an und mögen sogar fallweise eher peinlich gewesen sein, öffentlich artikuliert zu werden. So gehört etwa die Entscheidung zwischen diversen potentiellen Partnerinnen zwecks Heirat zumindest im späten Material zu den häufigeren Themen solcher Orakelfragen. Daher gab es als Variante - naheliegenderweise ausschließlich im privaten Bereich - die Möglichkeit, Fragen schriftlich zu stellen ${ }^{10}$. Dazu wurde die sehr knapp formulierte Frage auf ein Ostrakon geschrieben, das dann mit der Schrift nach unten auf den Prozessionsweg gelegt wurde. Auf der nach oben gedrehten Rückseite wurde ein einziges Symbol bzw. eine einzelne Hieroglyphe angebracht, die vielleicht dazu diente, das eigene Ostrakon wiederzuerkennen ${ }^{11}$. Vermutlich referiert die Orakelterminologie mit dem Begriffspaar „nehmen“ und „nicht nehmen“ auf die Beantwortung solcher Fragen, wobei jedoch im letzten Detail unklar bleiben muß, wie genau das Verfahren von statten ging.

Eine jüngere Weiterentwicklung zu dieser Variante stellt die Praxis dar, verschiedene Antwortoptionen auf die eigene Frage auf separate Papyruszettelchen zu schreiben. Ein Beispiel für einen besonders unsicheren Frager liefert etwa pCarlsberg 429 aus Tebtynis ${ }^{12}$ :

«Horos, Sohn des Pasis, ist es, der sagt: Mein großer Herr, oh Soknebtynis! Wenn es zu meinem guten Geschick ist, mir keine Frau als Ehefrau zu erwerben von diesen zwei Frauen, die geschrieben sind in meinem Brief, möge man mir diesen Brief herausbringen! Das gute Geschick, es ist vor Soknebtynis! Geschrieben.»

Offenbar handelt es sich um den dritten Zettel, auf zwei anderen, heute verlorenen, stand jeweils der Name einer der beiden Frauen.

Man könnte nun vermuten, auch diese Briefchen seien der Prozession in den Weg gelegt worden. Allerdings geht aus den beteiligten Texten nichts dergleichen hervor. Ganz im Gegenteil,

7 Černý 1962, 43-46, Kruchten 1997.

8 LÜDDECKENS 1980, 1370-1373.

9 Blackman 1926, Schenke 1960, Sweeney 2008, Muhs 2009.

10 Černý 1935, Černý 1941, Černý 1972, Zauzich 2000, Valbelle/Husson 1998, Martin 2004, Quack 2008, JÖRDENS 2008a.

11 WeIss 2009.

12 ZaUZich 2000, 6-7, Pl. 2. 
teilweise heißt es in den erhaltenen Exemplaren, so auch in dem zitierten „Wenn es gut für mich ist, xy zu tun, dann laß mir diesen Zettel herausgebracht werden“. Dadurch zeigt sich deutlich, daß diese Ticketorakel eine mindestens in der spätesten Zeit vom Prozessionsorakel deutlich zu trennende Form der göttlichen Willenserkundung sind. Dies wird durch das eingangs zitierte Dekret gestützt, das ebenfalls die beiden als getrennte Optionen aufführt, wobei die Prozession erst in zweiter Linie genannt wird. Es paßt weiterhin zum quantitativen Befund an Originalquellen, der zeigt, daß die ab der 3. Zwischenzeit sporadisch nachweisbaren Ticketorakel in der griechischrömischen Zeit dominant sind ${ }^{13}$, während die im Neuen Reich dominanten Prozessionsorakel in den spätesten Epochen seltener dokumentiert sind ${ }^{14}$. Der Unterschied zeigt sich auch in der Formulierung. Im Neuen Reich waren die meisten Orakelfragen als reale Frage „Ist xy der Fall?“ bzw. „Ist xy gut?“ formuliert, d.h. ein simples Nicken der Barke beim Passieren des betreffenden Ostrakons hätte ,ja“ bedeutet, in der Spätzeit hingegen lautet die Formulierung wie erwähnt „Wenn es gut für mich ist, xy zu tun, dann laß mir diesen Zettel herausgebracht werden“.

Die jetzt als Konditionalsätze formulierten Texte stehen auch nicht mehr auf Ostraka, sondern auf Papyruszetteln, die sorgfältig zusammengefaltet und damit für die Priester, die das Orakel durchführten, gar nicht lesbar waren. Der Gott mußte sich nun zwischen mindestens zwei auch physisch vorhandenen Antwortoptionen entscheiden. Daß beide Orakelformen auch durchaus nebeneinander bestehen können, ist sinnvoll, da es auch Fragen unterschiedlicher Komplexität gibt. Dies wird auch an dem eingangs zitierten Dekret deutlich, das einerseits von „in die Gegenwart der Gottheit legen“, d.h. vermutlich im Tempel, andererseits von „Prozessionen“" spricht ${ }^{15}$.

Aus griechisch-römischer Zeit gibt es auch einen Befund einer Statue, die in ihrem Inneren ein Rohr aufwies, das, so die gängige Interpretation, zur Erteilung eines gesprochenen Orakelspruches verwendet worden $\operatorname{sei}^{16}$. Aus älterer Zeit ist derlei nicht bekannt, so daß in Fällen, wo Texte davon sprechen, der Gott habe etwas "gesagt" ${ }^{17}$, fallweise unklar bleiben muß, ob hier wirklich eine Lautäußerung sprachlicher Art oder doch eher nur das Zustimmen zu einer entsprechenden schriftlichen oder mündlichen Formulierung durch eine Bewegung gemeint ist.

Klar ist in jedem Fall, daß es noch weitere Techniken bzw. Varianten bereits in älterer Zeit gegeben haben muß, da sich gelegentlich Hinweise darauf finden. Besonders kurios ist etwa eine Stele betreffs eines mutmaßlichen Orakelentscheids über eine Landschenkung von 35 Aruren, auf der eine in einer Kiste verborgene bzw. gerade daraus hervorkommende Frau vor den beteiligten Göttern Amun und Chons dargestellt ist ${ }^{18}$. Wie das Verfahren genau ablief, muß leider unklar bleiben.

Mindestens seit der Spätzeit gaben nachweislich nicht nur Götterbilder Orakel, sondern auch lebende Gottheiten, d.h. Heilige Tiere, insbesondere der Apisstier in Memphis. Diese

13 Für frühe Beispiele s. Ryholt 1993, zum späten Material s. Naether 2009 und in extenso Naether 2010.

$14 \mathrm{Daß}$ sie dennoch immer noch existierten, belegen außer dem eingangs zitierten Dekret jedoch auch Beschreibungen bei Strabo und Diodor, s. ČERnÝ 1962, 47.

15 Zur historischen Entwicklung und dem damit einhergehenden Wandel des Orakelwesens s. ČERnÝ 1962. Für das noch in der Spätantike aktive Besorakel in Abydos s. Dunand 1997, Effland 2013, Effland, Effland 2013, 126-130.

16 LoukianofF 1936.

17 So etwa in Fischer-Elfert 1996 (vgl. dazu auch Quack 1997, 83-84). Das aus demselben Milieu stammende Fragenpaar Rуноцт 1993 zeigt aber gerade, daß es sich eben nicht um eine verbale Äußerung handelt.

18 Kuhlmann 1992. 
erfolgten ebenfalls über Bewegungen, die Frage, ob das Tier Futter vom Bittsteller annahm, ihm die Hand leckte u.ä. ${ }^{19}$. Auch auf solche Praktiken nehmen die schon erwähnten Personennamen vom Typ $\breve{C} t-h r-n-N N$ Bezug, denn die Wesenheit NN ist teilweise ein Tier, z.B. ein Ibis oder Pavian ${ }^{20}$.

Wie das Dekret des römischen Präfekten und einige noch spätere Dokumente zeigen, spielte das Orakelwesen in Ägypten bis zum Ende der traditionellen Religion eine bedeutende Rolle $^{21}$. Schwieriger ist die Frage seines Beginns. Die ältesten unbestreitbaren Belege stammen aus dem frühen Neuen Reich, doch wurde immer wieder auf mögliche ältere Fälle verwie$\operatorname{sen}^{22}$. Wie erwähnt sind Prozessionsfeste mindestens ab dem Neuen Reich nachweislich der wichtigste Typ großer religiöser Volksfeste und ein wichtiger Rahmen für Orakelbefragungen gewesen. Hinweise auf entsprechende Veranstaltungen finden sich nun aber bereits aus dem Alten Reich, etwa im Wadi Hillal in Elkab, wohin regelmäßige Auszüge zu Ehren der Göttin Nechbet führten ${ }^{23}$. Ob diese allerdings bereits die Form der v.a. aus dem Theben des Neuen Reichs bekannten Prozessionsfeste hatten, läßt sich im Detail nicht sagen, auch nicht, ob es dabei zu Orakelbefragungen kam. Die Frage muß derzeit offenbleiben. Verf. tendiert jedoch zu der Annahme, daß es Orakel in diesem oder anderem Rahmen bereits vor dem Neuen Reich gegeben haben dürfte.

Wie bereits angedeutet, wurden sie quer durch alle Schichten der Gesellschaft konsultiert. Im staatlichen Bereich dienten sie etwa zur Legitimation von Feldzügen ${ }^{24}$, fallweise, wie beim Regierungsantritt der Hatschepsut als Pharao, sogar als Legitimation ihrer Herrschaft als solcher $^{25}$. Auch die Besetzung von Ämtern z.B. in der Priesterschaft erfolgte manchmal durch ein Orakel, so etwa die Vergabe des Hohenpriesteramtes des Amun unter Ramses II. an Nebwenenef ${ }^{26}$.

Im ersten Falle ist recht deutlich, warum auf ein Orakel rekurriert wurde. Hatschepsuts Griff nach der Pharaonenkrone als Frau war zwar nicht der erste solche Fall, aber dennoch ohne Frage rechtfertigungsbedürftig. Die Tatsache, daß der Reichsgott Amun selbst dies gewünscht hatte, machte den Vorgang hingegen für die Zeitgenossen schlechterdings unhinterfragbar ${ }^{27}$.

Etwas komplexer ist die Lage bei der Ernennung Nebwenenefs in Ramses' erstem Regierungsjahr. Das Amt des Hohenpriesters des Amun-Re von Karnak war das höchste Priesteramt im Lande und folglich eine Schlüsselposition. Normalerweise wurden solche Ämter innerhalb der jeweiligen Familie oder jedenfalls in einem recht kleinen Personenkreis weitervererbt ${ }^{28}$. Folglich dürfte die thebanische Priesterelite große Hoffnungen gehegt haben. Doch, wie Ramses Nebwenenef in seiner Einsetzungsrede mitteilte:

19 Courcelle 1951, 216, von Lieven 1999, 91-92.

20 LÜdDECKENs 1980, 1371-1372, QuaEgebeur 1997, spez. 30-31.

21 Tatsächlich sogar darüber hinaus, denn die späte Form des Ticketorakels wurde noch in koptischer Zeit sogar in christlichen Klöstern praktiziert, s. ČERnÝ 1962, 47-48, Husson 1997.

22 Brunner 1956, 60 (allerdings nicht stichhaltiges Argument), Baines, Parkinson 1997.

23 Zumindest war der Auszug in die Wüste zu einem dortigen Tempel am Neujahrstag offenbar wesentlicher Teil des nur für Elkab belegten $\breve{c} s r-t 3$-Festes, s. Vandekerckhove, Müller-Wollermann 2001, 41-44, 47.

24 Helck 1957, 1545,16-1546,2, Beylage 2002, 29-37, 580-588, Klug 2002, 345-352, 569 (Konosso-Stele).

25 Lacau, Chevrier 1977, 100-101, kontrovers kommentiert durch Assmann 1987, 50 und Römer 1987. Neuedition bei Burgos/Larché 2006, 30-42.

26 ETHE 1907, RoEDER 1960, 219-220.

27 Später bedienen sich übrigens auch Männer dieses Instrumentes, s. JANSEN-WINkelN 1999.

28 Für diese und verschiedene andere Möglichkeiten, ein religiöses Amt zu erlangen, s. von LIEvEN (in Druck). 
«Ich meldete ihm (dem Amun) den gesamten Hofstaat und den Obersten Mund des Heeres. Ich wiederholte ihm die Gottesdiener der Götter und die Großen seines Hauses, als sie angesichts seiner standen. Aber er war mit keinem einzigen von ihnen zufrieden, außer als ich ihm deinen Namen sagte. Führe ihm vortreffliche Taten aus, da er dich ja gewünscht hat...»

Man würde erwarten, daß man in Theben nicht gerade begeistert war, daß ein Priester aus Dendara den Zuschlag erhielt, doch darf dabei nicht vergessen werden, daß die Priester, die das Orakel durchführten und vermutlich die Kultstatue bewegten, ja genau diejenigen waren, die von einer andersartigen Entscheidung am meisten profitiert hätten ${ }^{29}$. Dies sollte eine deutliche Warnung davor sein, beim Orakelwesen stets Priesterbetrug am Werke zu sehen ${ }^{30}$. Vermutlich waren hier in der Regel gruppenpsychologische Phänomene am Werke, die nicht der willentlichen Kontrolle und Manipulation der Beteiligten unterlagen, wie sie auch heute noch in religiösen Zusammenhängen vorkommen.

Dabei ist es keineswegs ein Gegenargument, daß in der als „Elephantineskandal“ bekannt gewordenen Anklageschrift gegen den $w^{c} b$-Priester Penanuqet diesem vorgeworfen wird, versucht zu haben, das Orakel des Gottes Chnum zu beeinflussen:

«Anklage betreffs der Ernennung des Bakenchons zum Priester des Chnum durch den Wesir Neferrenpet, worüber dieser Wab-Priester zu dem Wab-Priester Nebwenenef sagte: „Wir werden drei andere Wab-Priester einweihen und den Gott veranlassen, diesen Sohn eines Kaufmanns zu entlassen. "Er wurde verhört und es wurde festgestellt, daß er das wirklich gesagt hatte. ${ }^{31}$.

Ganz im Gegenteil, die Tatsache, daß es sich hierbei offenbar um ein Sakrileg gehandelt hat, spricht dafür, daß entsprechende Absprachen eben nicht übliche Praxis waren. Die Warnung in Kapitel 20 der Lehre des Amenemope „Verfälsche kein Orakel auf Papyrus, zerstöre nicht den Ratschluß des Gottes. Maße dir nicht die Macht des Gottes selbst an, als ob es Schai und Renenet (Schicksal und Glück) nicht gäbe. "32 richtet sich hingegen offenbar spezifisch an Schreiber, die das Ergebnis protokollierten, wobei die Fortsetzung des Textes keinen Zweifel daran läßt, daß es um juristische Orakel in Eigentumsfragen geht. Eine ähnliche Warnung findet sich schon in der Lehre des Ani an der bereits erwähnten Stelle, wo davon abgeraten wird, sich beim Prozessionsorakel an das Götterbild heranzudrängeln. Einer der Gründe dafür ist denn auch sicher, daß dadurch das Orakel verfälscht werden könnte. Hier ist die Sorge jedoch die Bewegung der Barke selbst, nicht das schriftliche Protokoll, wie das Verbot, beim Tragen helfen zu wollen, deutlich macht.

Dank der schriftlichen Protokolle sind wir über die Verfahrensweise im Allgemeinen ebenso wie über die individuellen Streitgegenstände recht gut im Bilde. Ein besonders interessanter Fall wird in pBM 10335 aus der 20. Dynastie überliefert. Der Text zeigt exemplarisch, daß

29 Eine literarische Gestaltung des Themas mit ganz ähnlicher Konstellation liefert übrigens die Erzählung vom „Kampf um die Pfründe des Amun“ (pSpiegelberg und Parallelen), s. SpIegelberg 1910, 13-45, Taf. I-XXII, Hoffmann, Quack 2007, 88-107.

30 Vgl. die wichtigen Ausführungen bei Traunecker 1997.

31 Gardiner 1948, 75,9-11, Peden 1994, 111 (pTurin 1887 recto 1,12-13).

32 LAISNEY 2007, 188-195, 352-354. 
man in derselben Angelegenheit mehrere Götter konsultieren konnte, in der Hoffnung, eine günstigere Entscheidung zu erhalten. Besonders pikant ist dabei, daß es sich bei den verschiedenen Göttern de facto lediglich um verschiedene Lokalformen derselben Gottheit, nämlich des Gottes Amun handelt. Der Text lohnt ein genaueres Zitat ${ }^{33}$ :

«Zweites Regierungsjahr, dritter Überschwemmungsmonat, erster Tag. Der Beauftragte Amunemuja rief den Amun $p 3$ hnn.ti während seines schönen Festes, des Opetfestes, um Beistand an. Er sagte: „Komm [mir] zu Hilfe, Amun $p 3$ hnn.ti, mein guter und geliebter Herr. Der Oberste Verwalter des Viehs für den Altar hatte mich nämlich im Innern der Stadt postiert, um seinen Speicher zu bewachen und die Abgaben für ihn einzutreiben. Man ist mittags zu mir gekommen und hat fünf Hemden aus glattem Stoff, die sich unter meiner Obhut befanden, gestohlen. Mein guter und geliebter Herr, wirst du ihren Diebstahl aufdecken?” Das bejahte der Gott nachdrücklich.

Da nannte ihm der Beauftragte Amunemuja alle Ortsbewohner. Bei dem Feldarbeiter Patjauemdiamun stimmte der Gott plötzlich zu und verriet: „Er ist derjenige, der sie gestohlen hat." Dazu bemerkte der Feldarbeiter Patjauemdiamun im Beisein Amuns: „Lüge! Derjenige, der sie gestohlen hat, das bin ich nicht.” Darüber war der Gott in höchstem Maße aufgebracht.

Erneuter Versuch des Feldarbeiters Patjauemdiamun: Er zog vor Amun $t 3 \check{s} n \mid . t$ und sagte: „Ich bin enttäuscht von meinem Gott, ich werde zu dem anderen gehen”; er hatte vorher fünf Opferbrote in seinen Vorhof gebracht. Aber der Gott stimmte bei ihm in der besagten Weise zu und urteilte: „Er ist derjenige, der sie gestohlen hat.” Dazu sagte der Feldarbeiter Patjauemdiamun: „Das ist gelogen.” Deshalb befahl der Gott: „Bringt ihn in Anwesenheit starker ${ }^{34}$ Zeugen vor Amun $b w-k n n^{35} . .^{36}$

Er stand erneut vor Amun p3 hn.ti während seines schönen Festes Choiak zwecks dritter [Gegenüberstellung]. Er rief: „Komm mir zu Hilfe, Amun p3 hn.ti, mein guter und geliebter Herr. Bin ich es etwa, der die Kleider gestohlen hat?” Das bestätigte der Gott nachdrücklich und sagte: „Er ist derjenige, der sie gestohlen hat.” Er führte ihn vor und verabreichte ihm in Anwesenheit der Ortsbewohner eine Bestrafung. Nun legte er vor dem Gott einen Eid bei unserem Herrn ab und sprach ... : „Ich bin derjenige, der sie gestohlen hat." Der Gott legte vor den Einwohnern des Ortes ein Zeugnis ab und sprach:

33 Dawson 1925, Blackman 1925, Kammerzell 1986-1991 mit Korrekturen bei von Lieven 1999, 81-82 (danach das Zitat hier).

34 So $(k n)$, nicht „viele“ $\left(* c_{S} 3\right)$, wie bei KammerZell 1986-1991. Das paßt auch besser zur Liste der Zeugen, die nur drei Namen umfaßt, die aber vermutlich drei lokale Autoritätspersonen gewesen sein dürften.

35 Vermutlich eine Kurzform für den Personennamen $B w-k n(n)-t w=f$. Es würde sich also bei dem „Amun des Buqen(en)tuef" um eine von einer Person dieses Namens gestiftete Kultstatue des Amun handeln, die Bezeich-

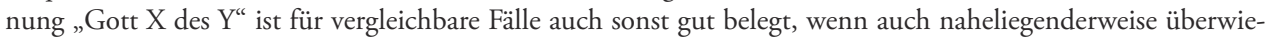
gend für Könige als Stifter. Im vorliegenden Falle ist diese Deutung besonders plausibel, da auch die anderen Amunepitheta mit spezifischen Kultorten verbundene Formen des Gottes bezeichnen ( $p 3$ hn.ti „,(von) der Vorhalle(?)“ (aufgrund des Stadtdeterminativs, das sonst nie belegt ist, ist die Übersetzung nicht absolut gesichert, vermutlich handelt es sich um die Bezeichnung eines ganzen Stadtviertels, denkbar wäre auch eine Übersetzung „(vom) Ortseingang" o.ä.), $t 3$ šnỉ.t „(von) der Umfassungsmauer"). Tatsächlich gab es in Deir el-Medineh eine Kapelle für Amun (sowie Hathor, Amenhotep I. und Ahmes-Nefertari), gestiftet von einem sç̣m- $\iota_{\breve{s}}$ Buqentuef (Bruyère 1948, 103, Pl. XV). Die kleine Kapelle des Buqentuef schließt dabei räumlich dicht an eine große Vorhalle ebenfalls kultischer Funktion an, was die Nähe zu Amun $p 3$ hn. $t i$, ja vielleicht gar Identität der beiden, im Text hinreichend erklären würde. Der Emendationsvorschlag von Kammerzell 1986-1991, 125, Anm. 1 a) ist damit nicht nur grammatisch problematisch, sondern auch sachlich hinfällig.

36 Es folgt eine Liste von drei namentlich benannten Zeugen mit ihren Amtstiteln. 


\begin{abstract}
„Seht, der Mann hat (den Diebstahl) der Kleider unseres Pharaos bestätigt, denn er sagte: „Sie befinden sich in meinem Besitz, aber ich will sie zurückgeben.” Der Verwalter Penherwer ... war es dann, der ihm ... hundert Schläge mit der Palmrippe verpaßte. Erneut nötigte er ihn, einen Eid bei unserem Herrn zu schwören: „Wenn ich meine Aussage wieder umstoße, dann sorgt dafür, daß ich dem Krokodil vorgeworfen werde.”...

Der Gott befahl, daß der Beauftragte Amunemuja folgenden Eid bei unserem Herrn schwören sollte: „Ich werde nicht eine Strafgebühr von ihm einziehen.“”
\end{abstract}

Daß derartige Verhandlungen sinnvollerweise in Gegenwart von Zeugen stattfinden sollten, liegt auf der Hand. Während der Fall des Kleiderdiebes Patjauemdiamun von eher geringer Bedeutung war, überliefert der als "Saite Oracle Papyrus”37 bekannte pBrooklyn 47.218 eine Orakelfrage betreffs eines Priesteramts. Der auf 651 v. Chr. datierte Papyrus ist über 5 $\mathrm{m}$ lang, mehr als $1 \mathrm{~m}$ davon nimmt eine leider stark zerstörte Vignette ein, die die Priester in Prozession mit dem Schrein des Amun-Re darstellt, denen eine Reihe hoher Würdenträger entgegengeht. Diese waren namentlich genannt und stimmen mit den wichtigsten der Personen überein, die das Dokument als Zeugen unterzeichneten, insgesamt 50 Personen. Es handelt sich hierbei um das prächtigste Orakelprotokoll aus dem Alten Ägypten überhaupt.

In der Regel waren die Fragen und Antworten an Orakel so klar formuliert, daß eine weitere Ausdeutung nicht mehr erforderlich war. Darin unterscheiden sich die traditionellen ägyptischen Orakel ganz grundlegend etwa von den griechischen ${ }^{38}$. Die ptolemäerzeitliche Statue des Platon, Sohn des Platon, eines hochrangigen Priesters zahlreicher Gottheiten, erwähnt jedoch Aktivitäten, die möglicherweise auf eine Orakelinterpretation hindeuten könnten ${ }^{39}$. Leider ist der Sachverhalt nicht hinreichend klar und ein Teil des Textes nicht erhalten.

Grundsätzlich konnte offenbar zumindest bei den Prozessionsfesten jeder Anfragen an das Orakel richten. Andererseits gibt es mehrere Texte, die zeigen, daß der Gott bei Orakeln, die nicht im Prozessionskontext stehen, quasi aus Höflichkeit erst befragt werden mußte, ob er überhaupt willens sei, Fragen zu beantworten, ein Faktum, das bisher in der Forschung weitgehend übersehen wurde. Besonders deutlich wird es in pCarlsberg 37a/b, einem auf $220 \mathrm{v}$. Chr. datierten Urkundenpaar aus Hawara, in dem der vergöttlichte Mensch „Teschnefer der Gott“ bezüglich eines Rechtsstreits um vermuteten Pfusch bei der Balsamierung des Ehemannes der Klägerin angerufen wird ${ }^{40}$. Bei genauer Lektüre der recht umfänglichen Texte fällt auf, daß vor der eigentlichen Orakelfrage erst angefragt wird, ob der Gott auch willens ist, überhaupt die Orakelfrage anzuhören. Zumindest als Möglichkeit wird auch einkalkuliert, daß er unwillig sein könnte. Als Frist zur Annahme sind anscheinend zehn Tage vorgesehen. Die Verpflichtung des Beklagten, nach erfolglosem Ablauf eines zehntägigen Anrufens der Gottheit eine Strafe zu bezahlen, kann eigentlich nur so gedeutet werden, daß keine Antwort auch eine Antwort ist, und zwar offenbar den Schuldspruch impliziert. Vermutlich steht dahinter der Gedanke, daß wenn der Gott den Frager keiner Reaktion würdigt, er schon seinen Grund haben muß. Im vorliegenden Falle erhielt der Frager jedoch gleich beim ersten Versuch eine positive Antwort und der gesamte Fall wurde zu den Akten gelegt.

37 PARKER 1962.

38 Generell zur Hermeneutik ägyptischer divinatorischer Techniken s. von LIEvEN (in Vorbereitung).

39 Coulon 2001.

40 LÜDdECKENs 1998, 37-54, Taf. 4, 5, QUACK 2008a, 382-384, ausführlich besprochen in vON LiEVEN 2007 s. $v$. $T \check{s}-n f r$. 
Tatsächlich ist dieses Prozedere kein Einzelfall, denn aus einigen älteren Texten ist derlei ebenfalls nachweisbar. So fragt Amenemuja in dem bereits zitierten pBM 10335 ja ebenfalls den Gott vor dem Beginn der Prozedur „Wirst du ihren Diebstahl aufdecken?“ Ähnliches findet sich auch auf der sog. „Stele der Verbannten“41, die ein Orakel staatstragender Bedeutung für die Nachwelt im Detail festhält. Dort wird einleitend sogar gefragt „Mein guter Herr! Gibt es eine Angelegenheit, die vor (dir) besprochen werden soll?”, d.h. der Gott selbst soll bekunden, ob es überhaupt ein Problem gibt. Letzterer Sonderfall ist freilich dadurch zu erklären, daß dieser Text aus der Epoche des sog. „thebanischen Gottesstaates des Amun“ (um 1000 v. Chr.) stammt ${ }^{42}$, in dem de jure der Gott Amun höchstpersönlich per Orakel als König herrschte und die gesamte Politik bestimmte, was de facto natürlich durch die jeweiligen Hohenpriester ausgeführt wurde, die auch die Orakel befragten. Diese wurden jedoch meist im Tempel selbst eingeholt, nicht notwendigerweise bei Prozessionen. Dementsprechend suggestiv fallen hier auch die Fragen aus. So heißt es in der Fortsetzung des Stelentextes:

«Er (d.h. der Hohepriester Mencheperre) trat erneut vor den großen Gott und sprach: „Mein guter Herr! Ist es die Angelegenheit deiner aufrührerischen Untertanen, denen du gezürnt hast und die sich jetzt in der Oase ${ }^{43}$ befinden, in die man verbannt?" Da stimmte der große Gott sehr stark zu. ... Wirst du heute auf meine Stimme hören und wirst du deinen aufrührerischen Untertanen, die du in die Oase verbannt hast, gnädig sein? Soll man sie nach Ägypten zurückholen?“ Da stimmte der große Gott sehr stark zu. ...»

Besonders interessant im Zusammenhang der Frage der Orakelwilligkeit eines Gottes ist pNevill, ein privater Gottesbrief der späten 20. Dyn., in dem sich der Schreiber beklagt, er habe dem Gott eine dringende Angelegenheit zur Entscheidung vorzutragen, dieser sei aber in seinem Allerheiligsten unerreichbar geblieben. Er müsse daher unbedingt in Prozession ausziehen, um sich der Sache anzunehmen. Der Bittsteller habe bislang keinerlei Nachricht von dem Gott, obwohl jemand seiner Stellung doch Befehle ausschicken könne ${ }^{44}$. Ob der ungeduldige Bittsteller, der explizit angibt, selbst keinen Zutritt zum Tempel zu haben und auch Schwierigkeiten gehabt zu haben, überhaupt jemanden zu finden, der als Bote den Brief dem Gott überbringen könne, mit seiner Anfrage Erfolg hatte, muß leider offenbleiben. Da Prozessionen mit einigem Aufwand verbunden waren und normalerweise nur zu Festen stattfanden, darf der Erfolg zumindest sehr bezweifelt werden.

Wie gesehen, rekurrierte sowohl der Staat, die Priester in Interna, als auch der kleine Mann auf der Straße auf Orakel. Wenn man nach den Gründen fragt, so ist zwischen den verschiedenen Ebenen zu differenzieren. Der Staat bediente sich des Instruments „Orakel“ sicherlich vielfach als legitimatorisches Mittel. Dennoch sollte auch bei Königen der Glaube an einen realen göttlichen Willensakt nicht a priori ausgeschlossen werden, wie dies in unserer generell agnostischen Zeit nur zu gerne geschieht.

Dies gilt auch für die beteiligten Priester und ihre internen Handlungen. Dennoch wird man sicher sagen dürfen, daß insbesondere im priesterlichen Milieu Orakelentscheidungen

41 von Beckerath 1968 (Stele Louvre C256).

42 Zu dieser Epoche s. Römer 1994.

43 Dachla oder Charga als Verbannungsort.

44 Barns 1949, Wente 1990, 219. 
als mehr oder minder „diplomatischer“ Weg zur Konsensfindung, etwa über die Vergabe von Ämtern, dienten. Mindestens in einem Fall wurde offenbar sogar zum Mittel einer Orakelbotschaft als akzeptabler Form der dienstlichen Rüge gegriffen ${ }^{45}$.

Dasselbe gilt sicher auch für die juristischen Orakel im Privatbereich. Es bot zweifelsohne weit weniger Stoff für sozialen Konflikt, wenn der Gott Amun einen Arbeiter des Diebstahls überführte, als wenn es seine Nachbarn taten. Daß fallweise der Beschuldigte dennoch nicht mit dem Ergebnis einverstanden war, liegt in der Natur der Sache. Leider unklar bleiben muß in allen diesen Fällen, ob der jeweils vom Gott schuldig Gesprochene auch wirklich der Täter war $^{46}$. Manchmal handelt es sich auch um Fälle, wo die Schuld eines Beklagten nicht oder nicht ohne weitere Probleme hätte geklärt werden können. In dem von Teschnefer, dem Gott entschiedenen Verfahren etwa hätte man die Mumie, um die es ging, beispielsweise wieder auswickeln müssen, was sicher als Sakrileg gegolten hätte und auch der Klägerin nicht recht gewesen wäre.

Verbleiben schließlich die Fälle privater Orakel, in denen es um Entweder-Oder-Entscheidungen in Handlungen des Privatlebens geht, wie etwa um das Unternehmen einer Geschäftsreise oder die Frage, welche von mehreren Frauen man besser heiraten solle. Wer je selbst längere Zeit über einer schwierigen Lebensentscheidung gebrütet hat, wird gewiß ohne Weiteres verstehen, warum man in einer solchen Situation auf den Gedanken kommen konnte, nicht auf die eigene beschränkte Weisheit zu bauen, sondern der Entscheidung eines weitaus weiseren Gottes zu vertrauen. Deutlicher kann die Opposition zwischen göttlicher Allmacht und menschlicher Ohnmacht nicht zum Ausdruck kommen.

\author{
Alexandra von Lieven \\ Freie Universität Berlin \\ Ägyptologisches Seminar \\ Fabeckstr. 23-25 \\ 14195 Berlin \\ alexandra.von.lieven@fu-berlin.de
}

45 S. die Ausführungen zu pDodgson in von Lieven 2004, 58-59.

46 Zweifel an der grundsätzlichen Gerechtigkeit von Orakelentscheidungen nährt etwa der Brief LRL 37 aus dem priesterlichen Milieu, s. QuACK 2008 b. 


\section{Bibliographie}

\section{el AMIr 1948}

M. el Amir, "The šḥH Ȟs of Apis at Memphis. A Season of Excavations at Mīt Rahīnah in 1941», Journal of Egyptian Archaeology 34 (1948), 51-56.

AsSMAnN 1987

J. Assmann, «Die Zeit Hatschepsuts und Thutmosis" III. in religionsgeschichtlicher Sicht", in A. Eggebrecht (Hg.), Agyptens Aufstieg zur Weltmacht, Mainz 1987, 47-55.

Baines, Parkinson I 997

J. Baines, R. B. Parkinson, "An Old Kingdom Record of an Oracle? Sinai Inscription 13", in J. van Dijk (Hg.), Essays on Ancient Egypt in Honour of Herman Te Velde (Egyptological Memoirs 1), Groningen 1997, 9-27.

BARNS I 949

J. Barns, "The Nevill Papyrus. A Late Ramesside Letter to an Oracle", Journal of Egyptian Archaeology 35 (1949), 69-71.

VON BECKERATH 1968

J. von Beckerath, «Die 'Stele der Verbannten' im Museum des Louvre», Revue d'Égyptologie 20 (1968), 7-36.

Beylage 2002

P. Beylage, Aufbau der königlichen Stelentexte vom Beginn der 18. Dynastie bis zur Amarnazeit (Ägypten und Altes Testament 54), Wiesbaden 2002.

BLACKMAN 1925

A.M. Blackman, "Oracles in Ancient Egypt I», Journal of Egyptian Archaeology 11 (1925), 249-255.

BLACKMAN 1926

A.M. Blackman, "Oracles in Ancient Egypt II», Journal of Egyptian Archaeology 12 (1926), 176-185.

BRUNNER 1956

H. Brunner, «Prozessionswunder im Mittleren Reich?», Zeitschrift für ägyptische Sprache und Altertumskunde 81 (1956), 60.

BruYÈre 1948

B. Bruyère, Rapports sur les fouilles de Deir el Médineh 1935-1940 (Fouilles de l'Institut Français d'Archéologie Orientale 20), Kairo 1948.

Burgos, Larché 2006

F. Burgos, F. LARChÉ, La chapelle rouge. Le sanctuaire de barque d'Hatshepsout I, Paris 2006.

\section{ČERnÝ 1935}

J. Černý, "Questions adressées aux oracles», Bulletin de l'Institut Français d'Archéologie Orientale 35 (1935), 41-58.

\section{ČERnÝ 1941}

J. Černý, "Nouvelle série de questions adressées aux oracles", Bulletin de l'Institut Français d'Archéologie Orientale 41 (1941), 13-25.

ČERNÝ 1962

J. Černý, "Egyptian Oracles», in: R. A. Parker, $A$ Saite Oracle Papyrus from Thebes, Providence 1962, 35-48.

ČERnÝ 1972

J. Černý, «Troisieme série de questions adressées aux oracles", Bulletin de l'Institut Français d'Archéologie Orientale 72 (1972), 49-69.

Cherpion, Corteggiani, Gout 2007

N. Cherpion, J.-P. Corteggiani, J.-Fr. Gout, Le tombeau de Pétosiris à Touna el-Gebel. Relevé photographique, Kairo 2007.

Coulon 2001

L. Coulon, "Quand Amon parle à Platon (La statue Caire JE 38033)», Revue d'Égyptologie 52 (2001), 85-125.

Courcelle 1951

P. Courcelle, "L'oracle d'Apis et l'oracle du jardin de Milan (Augustin, Conf. VIII 11,29)", Revue de l'histoire des religions 139/2 (1951) 216-231.

DAwson 1925

W. R. Dawson, «An oracle Papyrus. BM 10335», Journal of Egyptian Archaeology 11 (1925), 247-248.

Demichelis 2002

S. Demichelis, "La divination par l'huile à l'époque ramesside», in Y. Koenig (Hg.), La magie en Égypte, Actes du colloque organisé par le musée du Louvre les 29 et 30 septembre 2000 (La documentation Française), Paris 2002, 149-165.

DunAND 1997

F. Dunand, «La consultation oraculaire en Égypte tardive : l'oracle de Bès à Abydos», in J.-G. Heintz (Hg.), Oracles et prophéties dans l'antiquité, Actes du Colloque de Strasbourg, 15-17 juin 1995 (Travaux du Centre de Recherche sur le Proche-Orient et la Grèce antiques 15), Paris 1997, 65-84.

EFFLAND 2013

A. Effland, «»Bis auf den heutigen Tag begab sich kein Mensch mehr auf den Hügel von Abydos um zu opfern" - Zum Ende der Kulthandlungen in Umm elQa'āb”, in I. Gerlach, D. Raue (Hg.), Menschen - Kulturen - Traditionen 10: Sanktuar und Ritual. Heilige Plätze im archäologischen Befund (ForschungsCluster 4 Heiligtümer: Gestalt und Ritual, Kontinuität und Veränderung. Studien aus den Forschungsclustern 
des Deutschen Archäologischen Instituts), Rahden (Westf.) 2013, 75-82.

Effland, Effland 2013

U. Effland, A. Effland, Abydos. Tor zur ägyptischen Unterwelt, Darmstadt, Mainz 2013.

FISCHER-ELFERT 1996

H.-W. Fischer-Elfert, «Two Oracle Petitions Addressed to Horus-khau with Some Notes on the Oracular Amuletic Decrees (P. Berlin P. 8525 and P. 8526)", Journal of Egyptian Archaeology 82 (1996), 129-144.

FOUCART 1932

G. Foucart, Tombes thébaines (Mémoires publiés par les membres de l'Institut Français d'Archéologie Orientale du Caire 57), Kairo 1932.

Gardiner 1948

A. H. Gardiner, Ramesside Administrative Documents, Oxford 1948.

Helck 1957

W. Helck, Urkunden des ägyptischen Altertums IV. Urkunden der 18. Dynastie, Heft 19: Historische Inschriften Thutmosis' IV. und biographische Inschriften seiner Zeitgenossen, Berlin 1957.

Hoffmann, Quack 2007

F. Hoffmann, J.F. Quack, Anthologie der demotischen Literatur (Einführungen und Quellentexte zur Ägyptologie 4), Berlin 2007.

Husson 1997

G. Husson, «Les questions oraculaires chrétiennes d'Égypte. Continuités et changements», in B. Kramer (Hg.), Akten des 21. Internationalen Papyrologenkongresses, Berlin, 13. - 19. 8. 1995, Band 1 (Archiv für Papyrusforschung und verwandte Gebiete, Beihefte 3), Stuttgart 1997, 482-489.

JANSEN-WINKELN 1999

K. Jansen-Winkeln, "Die Wahl des Königs durch Orakel in der 20. Dynastie», Bulletin de la Société d'Égyptologie Genève 23 (1999), 51-61.

JÖRDENS 2008a

A. Jördens, "Orakelanfragen», in B. Janowski, G. Wilhelm e.a. (Hg.), Texte aus der Umwelt des Alten Testaments Neue Folge 4 Omina, Orakel, Rituale und Beschwörungen, Gütersloh 2008, 426-429.

JÖRDENS 2008b

A. Jördens, "Staatliches Verbot magischer Praktiken", in B. Janowski/G. Wilhelm e.a. (Hg.), Texte aus der Umwelt des Alten Testaments Neue Folge 4 Omina, Orakel, Rituale und Beschwörungen, Gütersloh 2008, 445.
Kammerzell 1986-1991

F. Kammerzell, «Aufklärung eines Diebstahls durch mehrfache Orakelbefragung», in O. Kaiser e.a. (Hg.), Texte aus der Umwelt des Alten Testaments II Orakel, Rituale. Bau- und Votivinschriften. Lieder und Gebete, Gütersloh 1986-1991, 123-126.

Karlshausen 2009

C. Karlshausen, Liconographie de la barque processionnelle divine en Egypte au Nouvel Empire (Orientalia Lovaniensia Analecta 182), Leuven 2009.

KLUG 2002

A. Klug, Königliche Stelen in der Zeit von Ahmose bis Amenophis III (Monumenta Aegyptiaca VIII), Turnhout 2002.

KRUCHTEN 1997

J.-M. Kruchten, «La terminologie de la consultation de l'oracle de l'Amon thébain à la IIIème Période Intermédiaire", in J.-G. Heintz (Hg.), Oracles et prophéties dans l'antiquité, Actes du Colloque de Strasbourg, 15-17 juin 1995 (Travaux du Centre de Recherche sur le Proche Orient et la Grèce antiques 15), Paris $1997,55-64$.

Kuhlmann 1992

K. P. Kuhlmann, «Ein eigentümliches Orakelverfahren aus der 22. Dynastie», in U. Luft (Hg.), The Intellectual Heritage of Egypt. Studies Presented to László Kákosy by Friends and Colleagues on the Occasion of his $60^{\text {th }}$ Birthday (Studia Aegyptiaca XIV), Budapest 1992, 367-372.

Lacau, Chevrier 1977

P. Lacau, H. Chevrier, Une chapelle d'Hatshepsout à Karnak I, Kairo 1977.

LANDESMUSEum WÜRTTEMBERg 2007

Landesmuseum Württemberg, Stuttgart (Hg.), Kata$\log$ Ägyptische Mumien. Unsterblichkeit im Land der Pharaonen, Mainz 2007.

LAISNEY 2007

V. P.-M. Laisney, L'enseignement d'Aménémope (Studia Pohl: Series Maior 19), Rom 2007.

VON LIEVEN 1999

A. von Lieven, "Divination in Ägypten", Altorientalische Forschungen 26 (1999) 77-126.

VON LIEVEN 2004

A. von Lieven, "Kinder, Schreiber, Könige - Vergöttlichte Menschen im Alten Ägypten. Ein Arbeitsbericht", Mitteilungen der Berliner Gesellschaft für Anthropologie, Ethnologie und Urgeschichte 25 (2004), 47-61.

VON LIEVEN 2007

A. von Lieven, Heiligenkult und Vergöttlichung im 
Alten Ägypten, Habilitationsschrift Berlin 2007 (Publikation in Vorbereitung).

von Lieven (in Druck)

A. von Lieven, " „Er war mit keinem einzigen von ihnen zufrieden, außer als ich ihm deinen Namen sagte." Religiöse Sukzession im Alten Ägypten», in A.B. Renger, M. Witte (Hg.), Sukzession in Religionen, (in Druck).

VON LIEVEN (in Vorbereitung)

A. von Lieven, «Divine Will and Human Decisionmaking. Divinatory Techniques in Ancient Egypt and their Hermeneutics», (Artikel in Vorbereitung).

LoukianofF 1936

G. Loukianoff, "Une statue parlante ou oracle du dieu Ré-Harmakhis», Annales du Service des Antiquités de l'Égypte 36 (1936), 187-193.

LÜDDECKENS 1980

E. Lüddeckens (Hg.), Demotisches Namenbuch, Wiesbaden 1980 .

LÜDDECKENS 1998

E. Lüddeckens, Demotische Urkunden aus Hawara (Verzeichnis der Orientalischen Handschriften in Deutschland Supplement 28), Stuttgart 1998.

MarTin 2004

C.J. Martin, «Questions to the Gods: Demotic Oracle Texts from Dimê», in F. Hoffmann, H.-J. Thissen (Hg.), Res severa verum gaudium. Festschrift für KarlTheodor Zauzich zum 65. Geburtstag am 8. Juni 2004 (Studia Demotica VI), Leuven, Paris, Dudley, MA 2004, 413-426.

Murs 2009

B.P. Muhs, "Oracular Property Decrees in their historical and chronological Context», in G.P.F. Broekman, R.J. Demarée, O.E. Kaper (Hg.), The Libyan Period in Egypt. Historical and cultural studies into the $21^{s t}-24^{\text {th }}$ dynasties. Proceedings of a conference at Leiden University, 25 - 27 October 2007 (Egyptologische Uitgaven 23), Leiden 2009, 265-275.

\section{NAEther 2009}

F. Naether, "Ticket-Orakel und Sortes Astrampsychi-Divinationsmethoden im griechisch-römischen Ägypten: ein Vorbericht», in G. Widmer/D. Devauchelle (Hg.), Actes du IX Congrès International des Études Démotiques, Paris, 31 Août-3 Septembre 2005 (Bibliothèque d'Étude 147), Kairo 2009, 253-265.

NAETHER 2010

F. Naether, Die Sortes Astrampsychi: Problemlösungsstrategien durch Orakel im römischen Ägypten (Orientalische Religionen in der Antike 3), Tübingen 2010.
PARKer 1962

R.A. Parker, A Saite Oracle Papyrus from Thebes, Providence 1962.

Peden 1994

A.J. Peden, The Reign of Ramesses IV, Warminster 1994.

QUACK 1994

J.F. Quack, Die Lehren des Ani. Ein neuägyptischer Weisheitstext in seinem kulturellen Umfeld (Orbis Biblicus et Orientalis 141), Freiburg (Schw.)/Göttingen 1994.

QUACK 1997

J.F. Quack, "Eine neue Deutung von pBerlin 8525», Göttinger Miszellen 159 (1997), 83-84.

QUACK 2006

J.F. Quack, "A Black Cat from the Right, and a Scarab on your Head. New Sources for Ancient Egyptian Divination", in K. Szpakowska (Hg.), Through a Glass Darkly: Magic, Dreams, and Prophecy in Ancient Egypt, Swansea 2006, 175-187.

QuAck 2008a

J.F. Quack, "Orakelanfragen», in B. Janowski/G. Wilhelm e.a. (Hg.), Texte aus der Umwelt des Alten Testaments Neue Folge 4 Omina, Orakel, Rituale und Beschwörungen, Gütersloh 2008, 371-372.

QuACK 2008b

J.F. Quack, «Henuttawis machtlose Unschuld. Zur Deutung von LRL 37», in Chr. Gallois, P. Grandet, L. Pantalacci (Hg.), Mélanges offerts à François Neveu par ses amis, élèves et collègues à l'occasion de son soixante-quinzième anniversaire (Bibliothèque d'Étude 145), Kairo 2008, 259-263.

Quaegebeur 1997

J. Quaegebeur, "L'appel au divin: le Bonheur des hommes mis dans la main des dieux", in J.-G. Heintz (Hg.), Oracles et prophéties dans l'antiquité, Actes du Colloque de Strasbourg, 15-17 juin 1995 (Travaux du Centre de Recherche sur le Proche-Orient et la Grèce antiques 15), Paris 1997, 15-34.

RITNER 1993

R. K. Ritner, The Mechanics of Ancient Egyptian Magical Practice (Studies in Ancient Oriental Civilization 54), Chicago 1993.

RoEDer 1960

G. Roeder, Kulte, Orakel und Naturverehrung im Alten Ägypten, Die ägyptische Religion in Texten und Bildern, Zürich/Stuttgart 1960.

RÖMER 1987

M. Römer, «Ist der Text auf den Blöcken 222/35/184 der Chapelle rouge ein Zeugnis für eine neue „Di- 
mension erfahrbarer Gottesnähe“ (Assmann)?», Göttinger Miszellen 99 (1987), 31-34.

RÖMER 1994

M. Römer, Studien zur Gottes- und Priesterherrschaft in Ägypten am Ende des Neuen Reiches (Ägypten und Altes Testament 21), Wiesbaden 1994.

RYHOLT 1993

K. Ryholt, «A Pair of Oracle Petitions Addressed to Horus-of-the-Camp", Journal of Egyptian Archaeology 79 (1993), 189-198.

SCHENKE 1960

H.-M. Schenke, Die Orakel im Alten Ägypten, Dissertation Berlin 1960.

SETHe 1907

K. Sethe, «Die Berufung eines Hohenpriesters des Amun unter Ramses II.», Zeitschrift für ägyptische Sprache und Altertumskunde 44 (1907), 30-35.

SPIEgeLberg 1910

W. Spiegelberg, Der Sagenkreis des Königs Petubastis nach dem Straßburger demotischen Papyrus sowie den Wiener und Pariser Bruchstücken (Demotische Studien 3), Leipzig 1910.

SWEENEY 2008

D. Sweeney, "Gender and Oracular Practice in Deir el-Medîna", Zeitschrift für ägyptische Sprache und Altertumskunde 135 (2008), 154-164.

Traunecker 1997

C. Traunecker, «L'appel au divin : la crainte des dieux et les serments de temple», in J.-G. Heintz (Hg.), Oracles et prophéties dans l'antiquité, Actes du Colloque de Strasbourg, 15-17 juin 1995 (Travaux du Centre de Recherche sur le Proche-Orient et la Grèce antiques 15), Paris 1997, 35-54.

Valbelle, Husson 1998

D. Valbelle, G. Husson, «Les Questions oraculaires d'Égypte. Histoire de la recherche, nouveautés et perspectives», in W. Clarysse (Hg.), Egyptian Religion: The Last Thousand Years. Studies dedicated to the memory of Jan Quaegebeur 2 (Orientalia Lovaniensia Analecta 85), Leuven 1998, 1055-1071.

VANDEKerckhove, Müller-Wollermann 2001

H. Vandekerckhove, R. Müller-Wollermann, Die Felsinschriften des Wadi Hilâl (Elkab VI), Turnhout 2001.

WEISS 2009

L. Weiss, "Markings on oracle ostraca from Deir el Medina - Conflicting interpretations», in B.J.J. Haring, O.E. Kaper (Hg.), Pictograms or pseudo script? Non-textual identity marks in practical use in ancient Egypt and elsewhere. Proceedings of a conference in Leiden, 19 - 20 December 2006 (Egyptologische Uitgaven 25) Leiden 2009, 221-230.

WENTE 1990

E. F. Wente, Letters From Ancient Egypt, Atlanta 1990.

\section{ZAUZICH 2000}

K.-Th. Zauzich, "Die demotischen Orakelfragen eine Zwischenbilanz», in P.J. Frandsen, K. Ryholt (Hg.), A Miscellany of Demotic Texts and Studies (The Carlsberg Papyri 3, Carsten Niebuhr Institute Publications 22), Copenhagen 2000, 1-25, Pl. 1-3. 